\title{
Classification of Exact Solutions for Generalized Form of $K(m, n)$ Equation
}

\author{
Hasan Bulut \\ Department of Mathematics, Faculty of Science, Firat University, 23119 Elazig, Turkey \\ Correspondence should be addressed to Hasan Bulut; hbulut@firat.edu.tr
}

Received 24 May 2013; Revised 1 August 2013; Accepted 18 August 2013

Academic Editor: Santanu Saha Ray

Copyright (C) 2013 Hasan Bulut. This is an open access article distributed under the Creative Commons Attribution License, which permits unrestricted use, distribution, and reproduction in any medium, provided the original work is properly cited.

The classification of exact solutions, including solitons and elliptic solutions, to the generalized $K(m, n)$ equation by the complete discrimination system for polynomial method has been obtained. From here, we find some interesting results for nonlinear partial differential equations with generalized evolution.

\section{Introduction}

In science and engineering applications, it is often very difficult to obtain analytical solutions of partial differential equations. Recently, many exact solutions of partial differential equations have been examined by the use of trial equation method. Also there are a lot of important methods that have been defined such as Hirota method, tanh-coth method, sine-cosine method, the trial equation method, and the extended trial equation method [1-15] to find exact solutions to nonlinear partial differential equations. There are a lot of nonlinear evolution equations that are solved by the use of various mathematical methods. Soliton solutions, singular solitons, and other solutions have been found by using these approaches. These obtained solutions have been encountered in various areas of applied mathematics and are very important.

In Section 2, we introduce an extended trial equation method for nonlinear evolution equations with higher order nonlinearity. In Section 3, as applications, we procure some exact solutions to nonlinear partial differential equations such as the generalized form of $K(m, n)$ equation [16-18]:

$$
\left(q^{l}\right)_{t}+a q^{m} q_{x}+b\left(q^{n}\right)_{x x x}=0,
$$

where $a, b \in R$ are constants since $l, m$, and $n \in Z^{+}$. Here, the first term is the generalized evolution term, while the second term represents the nonlinear term and the third term is the dispersion term. This equation is the generalized form of the KdV equation, where, in particular, the case $l=m=$ $n=1$ leads to the KdV equation. The Korteweg de Vries equation is one of the most important equations in applied mathematics and physics. There have been several kinds of solutions, such as compactons, that are studied in the context of $K(m, n)$ equation, for various situations. We now offer a more general trial equation method for discussion as follows.

\section{The Extended Trial Equation Method}

Step 1. For a given nonlinear partial differential equation

$$
P\left(u, u_{t}, u_{x}, u_{x x}, \ldots\right)=0
$$

take the general wave transformation

$$
u\left(x_{1}, x_{2}, \ldots, x_{N}, t\right)=u(\eta), \quad \eta=\lambda\left(\sum_{j=1}^{N} x_{j}-c t\right),
$$

where $\lambda \neq 0$ and $c \neq 0$. Substituting (3) into (2) yields a nonlinear ordinary differential equation:

$$
N\left(u, u^{\prime}, u^{\prime \prime}, \ldots\right)=0 .
$$

Step 2. Take the finite series and trial equation as follows:

$$
u=\sum_{i=0}^{\delta} \tau_{i} \Gamma^{i},
$$


where

$$
\left(\Gamma^{\prime}\right)^{2}=\Lambda(\Gamma)=\frac{\Phi(\Gamma)}{\Psi(\Gamma)}=\frac{\xi_{\theta} \Gamma^{\theta}+\cdots+\xi_{1} \Gamma+\xi_{0}}{\zeta_{\epsilon} \Gamma^{\epsilon}+\cdots+\zeta_{1} \Gamma+\zeta_{0}} .
$$

Using (5) and (6), we can write

$$
\begin{gathered}
\left(u^{\prime}\right)^{2}=\frac{\Phi(\Gamma)}{\Psi(\Gamma)}\left(\sum_{i=0}^{\delta} i \tau_{i} \Gamma^{i-1}\right)^{2}, \\
u^{\prime \prime}=\frac{\Phi^{\prime}(\Gamma) \Psi(\Gamma)-\Phi(\Gamma) \Psi^{\prime}(\Gamma)}{2 \Psi^{2}(\Gamma)}\left(\sum_{i=0}^{\delta} i \tau_{i} \Gamma^{i-1}\right) \\
+\frac{\Phi(\Gamma)}{\Psi(\Gamma)}\left(\sum_{i=0}^{\delta} i(i-1) \tau_{i} \Gamma^{i-2}\right)
\end{gathered}
$$

where $\Phi(\Gamma)$ and $\Psi(\Gamma)$ are polynomials. Substituting these relations into (4) yields an equation of polynomial $\Omega(\Gamma)$ of $\Gamma$ :

$$
\Omega(\Gamma)=\varrho_{s} \Gamma^{s}+\cdots+\varrho_{1} \Gamma+\varrho_{0}=0 .
$$

According to the balance principle, we can find a relation of $\theta, \epsilon$, and $\delta$. We can calculate some values of $\theta, \epsilon$, and $\delta$.

Step 3. Letting the coefficients of $\Omega(\Gamma)$ all be zero will yield an algebraic equations system:

$$
\varrho_{i}=0, \quad i=0, \ldots, s .
$$

Solving this system, we will determine the values of $\xi_{0}, \ldots, \xi_{\theta} ; \zeta_{0}, \ldots, \zeta_{\epsilon}$; and $\tau_{0}, \ldots, \tau_{\delta}$.

Step 4. Reduce (6) to the elementary integral form

$$
\pm\left(\eta-\eta_{0}\right)=\int \frac{d \Gamma}{\sqrt{\Lambda(\Gamma)}}=\int \sqrt{\frac{\Psi(\Gamma)}{\Phi(\Gamma)}} d \Gamma .
$$

Using a complete discrimination system for polynomial to classify the roots of $\Phi(\Gamma)$, we solve (10) and obtain the exact solutions to (4). Furthermore, we can write the exact traveling wave solutions to (2), respectively.

\section{Application to the Generalized Form of $K(m, n)$ Equation}

In order to look for travelling wave solutions of (1), we make the transformation $q(x, t)=u(\eta), \eta=x-c t$, where $c$ is the wave speed. Therefore it can be converted to the ODE

$$
-c\left(u^{l}(\eta)\right)^{\prime}+\frac{a}{m+1}\left(u^{m+1}(\eta)\right)^{\prime}+b\left(u^{n}(\eta)\right)^{\prime \prime \prime}=0,
$$

where prime denotes the derivative with respect to $\eta$. Then, integrating this equation with respect to $\eta$ one time and setting the integration constant to zero, we obtain

$$
-c u^{l}(\eta)+\frac{a}{m+1} u^{m+1}(\eta)+b\left(u^{n}(\eta)\right)^{\prime \prime}=0 .
$$

Let $l=n$, applying balance and using the following transformation:

$$
u=v^{1 /(m-n+1)} \text {. }
$$

Equation (12) turns into the following equation:

$$
\begin{aligned}
& -c(m+1)(m+1-n)^{2} v^{2}+a(m+1-n)^{2} v^{3} \\
& +b n(m+1)(2 n-m-1)\left(v^{\prime}\right)^{2} \\
& +b n(m+1)(m+1-n) v v^{\prime \prime}=0 .
\end{aligned}
$$

Substituting (7) into (14) and using balance principle yield

$$
\theta=\epsilon+\delta+2
$$

After this solution procedure, we obtain the results as follows.

Case 1. If we take $\epsilon=0, \delta=1$, and $\theta=3$, then

$$
\begin{gathered}
\left(v^{\prime}\right)^{2}=\frac{\left(\tau_{1}\right)^{2}\left(\xi_{3} \Gamma^{3}+\xi_{2} \Gamma^{2}+\xi_{1} \Gamma+\xi_{0}\right)}{\zeta_{0}}, \\
v^{\prime \prime}=\frac{\tau_{1}\left(3 \xi_{3} \Gamma^{2}+2 \xi_{2} \Gamma+\xi_{1}\right)}{2 \zeta_{0}},
\end{gathered}
$$

where $\xi_{3} \neq 0$ and $\zeta_{0} \neq 0$. Respectively, solving the algebraic equation system (9) yields

$$
\begin{aligned}
& \xi_{0}=-\frac{\xi_{1}^{2}(3+3 m-5 n)(1+m+n)}{16 \xi_{2}(1+m-2 n)^{2}}, \\
& \xi_{1}=\xi_{1}, \quad \xi_{2}=\xi_{2}, \\
& \xi_{3}=-\frac{8 \xi_{2}^{2}(1+m-2 n)(1+m-n)}{\xi_{1}(1+m+n)^{2}}, \\
& \tau_{0}=\tau_{0}, \quad \tau_{1}=-\frac{4(1+m-2 n) \xi_{2} \tau_{0}}{(1+m+n) \xi_{1}}, \\
& \zeta_{0}=-\frac{b n \xi_{2}(1+m)}{a(1+m-n) \tau_{0}}, \\
& c=\frac{a n(5+5 m-7 n) \tau_{0}}{(1+m)(1+m-n)(1+m+n)} .
\end{aligned}
$$

Substituting these results into (6) and (10), we have 


$$
\pm\left(\eta-\eta_{0}\right)=\frac{A}{2} \int \frac{d \Gamma}{\sqrt{\Gamma^{3}-\frac{\xi_{1}(1+m+n)^{2}}{8 \xi_{2}(1+m-2 n)(1+m-n)} \Gamma^{2}-\frac{\xi_{1}^{2}(1+m+n)^{2}}{8 \xi_{2}^{2}(1+m-2 n)(1+m-n)} \Gamma+\frac{\xi_{1}^{3}(3+3 m-5 n)(1+m+n)^{3}}{128 \xi_{2}^{2}(1+m-2 n)^{3}(1+m-n)}}},
$$

where

$$
A=\sqrt{\frac{b n \xi_{1}(1+m)(1+m+n)^{2}}{2 a \xi_{2} \tau_{0}(1+m-n)^{2}(1+m-2 n)}} .
$$

Integrating (18), we obtain the solutions to (1) as follows:

$$
\begin{gathered}
\pm\left(\eta-\eta_{0}\right)=-\frac{A}{\sqrt{\Gamma-\alpha_{1}}}, \\
\pm\left(\eta-\eta_{0}\right)=\frac{A}{\sqrt{\alpha_{2}-\alpha_{1}}} \arctan \sqrt{\frac{\Gamma-\alpha_{2}}{\alpha_{2}-\alpha_{1}}}, \quad \alpha_{2}>\alpha_{1}, \\
\pm\left(\eta-\eta_{0}\right)=\frac{A}{\sqrt{\alpha_{1}-\alpha_{2}}} \ln \left|\frac{\sqrt{\Gamma-\alpha_{2}}-\sqrt{\alpha_{1}-\alpha_{2}}}{\sqrt{\Gamma-\alpha_{2}}+\sqrt{\alpha_{1}-\alpha_{2}}}\right|, \quad \alpha_{1}>\alpha_{2}, \\
\pm\left(\eta-\eta_{0}\right)=-\frac{A}{\sqrt{\alpha_{1}-\alpha_{3}}} F(\varphi, l), \quad \alpha_{1}>\alpha_{2}>\alpha_{3},
\end{gathered}
$$

where

$$
\begin{gathered}
F(\varphi, l)=\int_{0}^{\varphi} \frac{d \psi}{\sqrt{1-l^{2} \sin ^{2} \psi}}, \quad \varphi=\arcsin \sqrt{\frac{\Gamma-\alpha_{3}}{\alpha_{2}-\alpha_{3}}}, \\
l^{2}=\frac{\alpha_{2}-\alpha_{3}}{\alpha_{1}-\alpha_{3}} .
\end{gathered}
$$

Also $\alpha_{1}, \alpha_{2}$, and $\alpha_{3}$ are the roots of the polynomial equation

$$
\Gamma^{3}+\frac{\xi_{2}}{\xi_{3}} \Gamma^{2}+\frac{\xi_{1}}{\xi_{3}} \Gamma+\frac{\xi_{0}}{\xi_{3}}=0 .
$$

Substituting solutions (20) into (5) and (13), we have

$$
\begin{aligned}
& u(x, t) \\
& =\left[\tau_{0}+\tau_{1} \alpha_{1}\right. \\
& +\left(A ^ { 2 } \tau _ { 1 } \left(\left(x-\left(a n(5+5 m-7 n) \tau_{0}\right)\right.\right.\right. \\
& \\
& \quad \times((1+m)(1+m-n)(1+m+n))^{-1} \\
& \\
& \left.\left.\left.\left.\quad \times t-\eta_{0}\right)^{2}\right)^{-1}\right)\right]^{1 /(m-n+1)},
\end{aligned}
$$

$$
\begin{aligned}
& u(x, t)=\left[\tau_{0}+\tau_{1} \alpha_{1}+\tau_{1}\left(\alpha_{2}-\alpha_{1}\right)\right. \\
& \times \operatorname{sech}^{2}\left(\frac { \sqrt { \alpha _ { 2 } - \alpha _ { 1 } } } { A } \left(x-\left(\operatorname{an}(5+5 m-7 n) \tau_{0}\right)\right.\right. \\
& \times((1+m)(1+m-n) \\
& \times(1+m+n))^{-1} \\
& \left.\left.\left.\times t-\eta_{0}\right)\right)\right]^{1 /(m-n+1)} \\
& =\left[\tau_{0}+\tau_{1} \alpha_{1}+\tau_{1}\left(\alpha_{1}-\alpha_{2}\right)\right. \\
& \times \operatorname{cosech}^{2}\left(\frac { \sqrt { \alpha _ { 1 } - \alpha _ { 2 } } } { 2 A } \left(x-\left(\operatorname{an}(5+5 m-7 n) \tau_{0}\right)\right.\right. \\
& \times((1+m)(1+m-n) \\
& \times(1+m+n))^{-1} \\
& \left.\left.\left.\times t-\eta_{0}\right)\right)\right]^{1 /(m-n+1)}
\end{aligned}
$$

$u(x, t)$

$$
\begin{aligned}
=\left[\tau_{0}+\tau_{1} \alpha_{1}\right. & +\left(\tau_{1}\left(\alpha_{2}-\alpha_{1}\right)\right) \\
\times\left(\operatorname{sn}^{2}(\right. & \pm \frac{\sqrt{\alpha_{2}-\alpha_{1}}}{A} \\
& \times\left(x-\frac{a n(5+5 m-7 n) \tau_{0}}{(1+m)(1+m-n)(1+m+n)}\right.
\end{aligned}
$$$$
\left.\times t-\eta_{0}\right) \text {, }
$$$$
\left.\left.\left.\frac{\alpha_{1}-\alpha_{3}}{\alpha_{1}-\alpha_{2}}\right)\right)^{-1}\right]^{1 /(m-n+1)} \text {. }
$$

If we take $\tau_{0}=-\tau_{1} \alpha_{1}$ and $\eta_{0}=0$, then solutions (23) can reduce to rational function solution

$$
u(x, t)=\left(\frac{\widetilde{A}}{x-c t}\right)^{2 /(m-n+1)},
$$


1-soliton wave solution

$$
u(x, t)=\frac{\widetilde{B}}{\cosh ^{2 /(m-n+1)}(B(x-c t))},
$$

singular soliton solution

$$
u(x, t)=\frac{\widetilde{C}}{\sinh ^{2 /(m-n+1)}(C(x-c t))},
$$

and elliptic soliton solution

$$
u(x, t)=\frac{\widetilde{B}}{s n^{2 /(m-n+1)}(\varphi, l)},
$$

where $\widetilde{A}=A \sqrt{\tau_{1}}, \widetilde{B}=\left(\tau_{1}\left(\alpha_{2}-\alpha_{1}\right)\right)^{1 /(m-n+1)}, B=$ $\sqrt{\alpha_{2}-\alpha_{1}} / A, \widetilde{C}=\left(\tau_{1}\left(\alpha_{1}-\alpha_{2}\right)\right)^{1 /(m-n+1)}, C=\sqrt{\alpha_{1}-\alpha_{2}} / 2 A$, $\varphi= \pm\left(\sqrt{\alpha_{2}-\alpha_{1}} / A\right)(x-c t), l^{2}=\left(\alpha_{1}-\alpha_{3}\right) /\left(\alpha_{1}-\alpha_{2}\right)$, and $c=\operatorname{an}(5+5 m-7 n) \tau_{1} \alpha_{1} /(1+m)(1+m-n)(1+m+n)$. Here, $\widetilde{B}$ and $\widetilde{C}$ are the amplitudes of the solitons, while $B$ and $C$ are the inverse widths of the solitons and $c$ is the velocity. Thus, we can say that the solitons exist for $\tau_{1}>0$.

Remark 1. If we choose the corresponding values for some parameters, solution (25) is in full agreement with solution (21) mentioned in [17].

Case 2. If we take $\epsilon=0, \delta=2$, and $\theta=4$, then

$$
\left(v^{\prime}\right)^{2}=\frac{\left(\tau_{1}+2 \tau_{2} \Gamma\right)^{2}\left(\xi_{4} \Gamma^{4}+\xi_{3} \Gamma^{3}+\xi_{2} \Gamma^{2}+\xi_{1} \Gamma+\xi_{0}\right)}{\zeta_{0}},
$$

where $\xi_{4} \neq 0$ and $\zeta_{0} \neq 0$. Respectively, solving the algebraic equation system (9) yields

$$
\begin{gathered}
\xi_{0}=\xi_{0}, \quad \xi_{1}=\xi_{1}, \quad \xi_{2}=\frac{\xi_{1}^{2}}{3 \xi_{0}}, \quad \xi_{3}=\frac{\xi_{1}^{3}}{24 \xi_{0}^{2}}, \\
\xi_{4}=\frac{\xi_{1}^{4}}{576 \xi_{0}^{3}}, \quad \zeta_{0}=-\frac{b n(m+1)(m+n+1) \xi_{1}^{3}}{24 a(m-n+1)^{2} \xi_{0}^{2} \tau_{1}}, \\
\tau_{0}=\frac{2 \xi_{0} \tau_{1}}{\xi_{1}}, \quad \tau_{1}=\tau_{1}, \quad \tau_{2}=\frac{\xi_{1} \tau_{1}}{12 \xi_{0}}, \\
c=-\frac{2 a n \xi_{0} \tau_{1}}{(m+1)(m+n+1) \xi_{1}} .
\end{gathered}
$$

Substituting these resultss into (6) and (10), we get

$$
\begin{aligned}
& \pm\left(\eta-\eta_{0}\right) \\
& =2 A_{1} \int((d \Gamma) \\
& \times\left(\Gamma^{4}+\left(\frac{24 \xi_{0}}{\xi_{1}}\right) \Gamma^{3}+\left(\frac{192 \xi_{0}^{2}}{\xi_{1}^{2}}\right) \Gamma^{2}\right. \\
& \left.\left.+\left(\frac{576 \xi_{0}^{3}}{\xi_{1}^{3}}\right) \Gamma+\left(\frac{576 \xi_{0}^{4}}{\xi_{1}^{4}}\right)\right)^{-1 / 2}\right),
\end{aligned}
$$

where $A_{1}=\sqrt{-6 b n \xi_{0}(1+m)(1+m+n) / a \xi_{1} \tau_{1}(1+m-n)^{2}}$. Integrating (30), we obtain the solutions to (1) as follows:

$$
\begin{gathered}
\pm\left(\eta-\eta_{0}\right)=-\frac{2 A_{1}}{\Gamma-\alpha_{1}}, \\
\pm\left(\eta-\eta_{0}\right)=\frac{4 A_{1}}{\alpha_{1}-\alpha_{2}} \sqrt{\frac{\Gamma-\alpha_{2}}{\Gamma-\alpha_{1}}}, \quad \alpha_{1}>\alpha_{2}, \\
\pm\left(\eta-\eta_{0}\right)=\frac{2 A_{1}}{\alpha_{1}-\alpha_{2}} \ln \left|\frac{\Gamma-\alpha_{1}}{\Gamma-\alpha_{2}}\right|, \\
=\frac{\left.\eta_{0}\right) \quad \alpha_{1}}{\sqrt{\left(\alpha_{1}-\alpha_{2}\right)\left(\alpha_{1}-\alpha_{3}\right)}} \\
\quad \times \ln \left|\frac{\sqrt{\left(\Gamma-\alpha_{2}\right)\left(\alpha_{1}-\alpha_{3}\right)}-\sqrt{\left(\Gamma-\alpha_{3}\right)\left(\alpha_{1}-\alpha_{2}\right)}}{\sqrt{\left(\Gamma-\alpha_{2}\right)\left(\alpha_{1}-\alpha_{3}\right)}+\sqrt{\left(\Gamma-\alpha_{3}\right)\left(\alpha_{1}-\alpha_{2}\right)}}\right| \\
\quad \pm\left(\eta-\eta_{0}\right)=\frac{\alpha_{1}>\alpha_{2}>\alpha_{3}}{\sqrt{\left(\alpha_{1}-\alpha_{4}\right)\left(\alpha_{2}-\alpha_{3}\right)}} F(\varphi, l),
\end{gathered}
$$

where

$$
\begin{aligned}
& \varphi_{1}=\arcsin \sqrt{\frac{\left(\Gamma-\alpha_{2}\right)\left(\alpha_{1}-\alpha_{4}\right)}{\left(\Gamma-\alpha_{1}\right)\left(\alpha_{2}-\alpha_{4}\right)}}, \\
& l_{1}^{2}=\frac{\left(\alpha_{1}-\alpha_{3}\right)\left(\alpha_{2}-\alpha_{4}\right)}{\left(\alpha_{2}-\alpha_{3}\right)\left(\alpha_{1}-\alpha_{4}\right)} .
\end{aligned}
$$

Also $\alpha_{1}, \alpha_{2}, \alpha_{3}$, and $\alpha_{4}$ are the roots of the polynomial equation

$$
\Gamma^{4}+\frac{\xi_{3}}{\xi_{4}} \Gamma^{3}+\frac{\xi_{2}}{\xi_{4}} \Gamma^{2}+\frac{\xi_{1}}{\xi_{4}} \Gamma+\frac{\xi_{0}}{\xi_{4}}=0 .
$$

Substituting solutions (31) into (5) and (13), we have

$$
\begin{aligned}
& u(x, t) \\
& =\left[\tau_{0}+\tau_{1} \alpha_{1} \pm\left(2 \tau_{1} A_{1}\right)\right. \\
& \quad \times\left(x+\frac{2 a n \xi_{0} \tau_{1}}{(m+1)(m+n+1) \xi_{1}} t-\eta_{0}\right)^{-1}
\end{aligned}
$$




$$
\begin{aligned}
+\tau_{2}\left(\alpha_{1} \pm\left(2 A_{1}\right)\right. \\
+\left(x+\frac{2 a n \xi_{0} \tau_{1}}{(m+1)(m+n+1) \xi_{1}}\right. \\
\left.\left.\left.\quad \times t-\eta_{0}\right)^{-1}\right)^{2}\right]^{1 /(m-n+1)},
\end{aligned}
$$

$u(x, t)$

$$
\begin{aligned}
& =\left[\tau_{0}+\tau_{1} \alpha_{1}\right. \\
& +\left(16 A_{1}^{2}\left(\alpha_{2}-\alpha_{1}\right) \tau_{1}\right) \\
& \times\left(16 A_{1}^{2}-\left[\left(\alpha_{1}-\alpha_{2}\right)\right.\right. \\
& \left.\left.\times\left(x+\frac{2 a n \xi_{0} \tau_{1}}{(m+1)(m+n+1) \xi_{1}} t-\eta_{0}\right)\right]^{2}\right)^{-1} \\
& +\tau_{2}\left(\alpha_{1}+\left(16 A_{1}^{2}\left(\alpha_{2}-\alpha_{1}\right)\right)\right. \\
& \times\left(16 A_{1}^{2}-\left[\left(\alpha_{1}-\alpha_{2}\right)\right.\right. \\
& \times\left(x+\frac{2 a n \xi_{0} \tau_{1}}{(m+1)(m+n+1) \xi_{1}}\right. \\
& \left.\left.\left.\left.\times\left(-\eta_{0}\right)\right]^{2}\right)^{-1}\right)^{2}\right]^{1 /(m-n+1)}
\end{aligned}
$$

$u(x, t)$

$$
\begin{aligned}
& =\left[\tau_{0}+\tau_{1} \alpha_{2}+\left(\left(\alpha_{2}-\alpha_{1}\right) \tau_{1}\right)\right. \\
& \times\left(\operatorname { e x p } \left[\frac{\alpha_{1}-\alpha_{2}}{2 A_{1}}\right.\right. \\
& \left.\left.\times\left(x+\frac{2 a n \xi_{0} \tau_{1}}{(m+1)(m+n+1) \xi_{1}} t-\eta_{0}\right)\right]-1\right)^{-1} \\
& +\tau_{2}\left(\alpha_{2}+\left(\alpha_{2}-\alpha_{1}\right)\right. \\
& \times\left(\operatorname { e x p } \left[\frac{\alpha_{1}-\alpha_{2}}{2 A_{1}}\right.\right. \\
& \times\left(x+\frac{2 a n \xi_{0} \tau_{1}}{(m+1)(m+n+1) \xi_{1}}\right. \\
& \left.\left.\left.\left.\left.\times t-\eta_{0}\right)\right]-1\right)^{-1}\right)^{2}\right]^{1 /(m-n+1)},
\end{aligned}
$$

$$
\begin{aligned}
& u(x, t) \\
& =\left[\tau_{0}+\tau_{1} \alpha_{1}+\left(\left(\alpha_{1}-\alpha_{2}\right) \tau_{1}\right)\right. \\
& \times\left(\operatorname { e x p } \left[\frac{\alpha_{1}-\alpha_{2}}{2 A_{1}}\right.\right. \\
& \times\left(x+\left(2 a n \xi_{0} \tau_{1}\right)\right.
\end{aligned}
$$$$
\times\left((m+1)(m+n+1) \xi_{1}\right)^{-1}
$$$$
\left.\left.\left.\times t-\eta_{0}\right)\right]-1\right)^{-1}
$$$$
+\tau_{2}\left(\alpha_{1}+\left(\alpha_{1}-\alpha_{2}\right)\right.
$$$$
\times\left(\operatorname { e x p } \left[\frac{\alpha_{1}-\alpha_{2}}{2 A_{1}}\right.\right.
$$$$
\times\left(x+\left(2 a n \xi_{0} \tau_{1}\right)\right.
$$$$
\times\left((m+1)(m+n+1) \xi_{1}\right)^{-1}
$$$$
\left.\left.\left.\left.\left.\times t-\eta_{0}\right)\right]-1\right)^{-1}\right)^{2}\right]^{1 /(m-n+1)}
$$

$u(x, t)$

$$
\begin{gathered}
=\left[\begin{array}{l}
\tau_{0}+\tau_{1} \alpha_{1}-\left(2\left(\alpha_{1}-\alpha_{2}\right)\left(\alpha_{1}-\alpha_{3}\right) \tau_{1}\right) \\
\quad \times\left(2 \alpha_{1}-\alpha_{2}-\alpha_{3}+\left(\alpha_{3}-\alpha_{2}\right)\right.
\end{array}\right. \\
\end{gathered}
$$$$
\times \cosh \left[\frac{\sqrt{\left(\alpha_{1}-\alpha_{2}\right)\left(\alpha_{1}-\alpha_{3}\right)}}{2 A_{1}}\right.
$$$$
\times\left(x+\frac{2 a n \xi_{0} \tau_{1}}{(m+1)(m+n+1) \xi_{1}}\right.
$$$$
\left.\left.\left.\times t-\eta_{0}\right)\right]\right)^{-1}
$$

$$
\begin{gathered}
+\tau_{2}\left(\begin{array}{l}
\alpha_{1}-\left(2\left(\alpha_{1}-\alpha_{2}\right)\left(\alpha_{1}-\alpha_{3}\right)\right) \\
\times\left(2 \alpha_{1}-\alpha_{2}-\alpha_{3}+\left(\alpha_{3}-\alpha_{2}\right)\right.
\end{array}\right.
\end{gathered}
$$




$$
\begin{aligned}
\times \cosh \left[\frac{\sqrt{\left(\alpha_{1}-\alpha_{2}\right)\left(\alpha_{1}-\alpha_{3}\right)}}{2 A_{1}}\right. \\
\times\left(x+\frac{2 a n \xi_{0} \tau_{1}}{(m+1)(m+n+1) \xi_{1}}\right. \\
\left.\left.\left.\left.\left.\times t-\eta_{0}\right)\right]\right)^{-1}\right)^{2}\right]^{1 /(m-n+1)}
\end{aligned}
$$

$u(x, t)$

$$
=\left[\tau_{0}+\tau_{1} \alpha_{2}+\left(\left(\alpha_{1}-\alpha_{2}\right)\left(\alpha_{4}-\alpha_{2}\right) \tau_{1}\right)\right.
$$$$
\times\left(\left(\alpha_{1}-\alpha_{4}\right)\right.
$$$$
\times \operatorname{sn}^{2}\left(\frac{\sqrt{\left(\alpha_{1}-\alpha_{3}\right)\left(\alpha_{2}-\alpha_{4}\right)}}{4 A_{1}}\right.
$$$$
\times\left(x+\frac{2 a n \xi_{0} \tau_{1}}{(m+1)(m+n+1) \xi_{1}}\right.
$$$$
\left.\times t-\eta_{0}\right)
$$$$
\left.\frac{\left(\alpha_{2}-\alpha_{3}\right)\left(\alpha_{1}-\alpha_{4}\right)}{\left(\alpha_{1}-\alpha_{3}\right)\left(\alpha_{2}-\alpha_{4}\right)}\right)
$$$$
\left.+\alpha_{4}-\alpha_{2}\right)^{-1}
$$$$
+\tau_{2}\left(\alpha_{2}+\left(\left(\alpha_{1}-\alpha_{2}\right)\left(\alpha_{4}-\alpha_{2}\right) \tau_{1}\right)\right.
$$$$
\begin{array}{r}
\times\left(\alpha_{1}-\alpha_{4}\right) \\
\times \operatorname{sn}^{2}\left(\frac{\sqrt{\left(\alpha_{1}-\alpha_{3}\right)\left(\alpha_{2}-\alpha_{4}\right)}}{4 A_{1}}\right.
\end{array}
$$$$
\times\left(x+\frac{2 a n \xi_{0} \tau_{1}}{(m+1)(m+n+1) \xi_{1}}\right.
$$

$$
\left.\times t-\eta_{0}\right)
$$$$
\left.\frac{\left(\alpha_{2}-\alpha_{3}\right)\left(\alpha_{1}-\alpha_{4}\right)}{\left(\alpha_{1}-\alpha_{3}\right)\left(\alpha_{2}-\alpha_{4}\right)}\right)
$$$$
\left.\left.\left.+\alpha_{4}-\alpha_{2}\right)^{-1}\right)^{2}\right]^{1 /(m-n+1)} .
$$

For simplicity, if we take $\eta_{0}=0$, then we can write solutions (34) as follows:

$$
u(x, t)=\left[\sum_{i=0}^{2} \tau_{i}\left(\alpha_{1} \pm \frac{2 A_{1}}{x-c t}\right)^{i}\right]^{1 /(m-n+1)},
$$

$$
u(x, t)
$$$$
=\left[\sum _ { i = 0 } ^ { 2 } \tau _ { i } \left(\alpha_{1}+\left(16 A_{1}^{2}\left(\alpha_{1}-\alpha_{2}\right)\right)\right.\right.
$$$$
\left.\left.\times\left(16 A_{1}^{2}-\left[\left(\alpha_{1}-\alpha_{2}\right)(x-c t)\right]^{2}\right)^{-1}\right)^{i}\right]^{1 /(m-n+1)},
$$$$
u(x, t)=\left[\sum_{i=0}^{2} \tau_{i}\left(\alpha_{2}+\frac{\alpha_{2}-\alpha_{1}}{\exp \left[B_{1}(x-c t)\right]-1}\right)^{i}\right]^{1 /(m-n+1)}
$$$$
u(x, t)=\left[\sum_{i=0}^{2} \tau_{i}\left(\alpha_{1}+\frac{\alpha_{1}-\alpha_{2}}{\exp \left[B_{1}(x-c t)\right]-1}\right)^{i}\right]^{1 /(m-n+1)}
$$$$
u(x, t)=\left[\sum _ { i = 0 } ^ { 2 } \tau _ { i } \left(\alpha_{1}-\left(2\left(\alpha_{1}-\alpha_{2}\right)\left(\alpha_{1}-\alpha_{3}\right)\right)\right.\right.
$$

$$
\times\left(2 \alpha_{1}-\alpha_{2}-\alpha_{3}+\left(\alpha_{3}-\alpha_{2}\right)\right.
$$$$
\left.\left.\left.\times \cosh \left[C_{1}(x-c t)\right]\right)^{-1}\right)^{i}\right]^{1 /(m-n+1)},
$$

$$
\begin{gathered}
u(x, t)=\left[\sum _ { i = 0 } ^ { 2 } \tau _ { i } \left(\alpha_{2}+\left(\left(\alpha_{1}-\alpha_{2}\right)\left(\alpha_{4}-\alpha_{2}\right)\right)\right.\right. \\
\times\left(\left(\alpha_{1}-\alpha_{4}\right) s n^{2}(\varphi, l)\right. \\
\left.\left.\left.+\alpha_{4}-\alpha_{2}\right)^{-1}\right)^{i}\right]^{1 /(m-n+1)},
\end{gathered}
$$

where $B_{1}=\left(\alpha_{1}-\alpha_{2}\right) / 2 A_{1}, C_{1}=\sqrt{\left(\alpha_{1}-\alpha_{2}\right)\left(\alpha_{1}-\alpha_{3}\right)} / 2 A_{1}$, $\varphi_{1}=\left(\sqrt{\left(\alpha_{1}-\alpha_{3}\right)\left(\alpha_{2}-\alpha_{4}\right)} / 4 A_{1}\right)(x-c t), l_{1}^{2}=\left(\alpha_{2}-\alpha_{3}\right)\left(\alpha_{1}-\right.$ $\left.\alpha_{4}\right) /\left(\alpha_{1}-\alpha_{3}\right)\left(\alpha_{2}-\alpha_{4}\right)$, and $c=-2 a n \xi_{0} \tau_{1} /(m+1)(m+n+1) \xi_{1}$. Here, $A_{1}$ is the amplitude of the soliton, while $c$ is the velocity 
and $B_{1}$ and $C_{1}$ are the inverse widths of the solitons. Thus, we can say that the solitons exist for $\tau_{1}>0$.

Case 3. If we take $\epsilon=0, \delta=3$, and $\theta=5$, then

$$
\begin{aligned}
\left(v^{\prime}\right)^{2}= & \left(\tau_{1}+2 \tau_{2} \Gamma+3 \tau_{3} \Gamma^{2}\right)^{2} \\
& \times\left(\xi_{5} \Gamma^{5}+\xi_{4} \Gamma^{4}+\xi_{3} \Gamma^{3}+\xi_{2} \Gamma^{2}+\xi_{1} \Gamma+\xi_{0}\right) \\
& \times\left(\zeta_{0}\right)^{-1}
\end{aligned}
$$

where $\xi_{5} \neq 0$ and $\zeta_{0} \neq 0$. Respectively, solving the algebraic equation system (9) yields

$$
\begin{aligned}
& \xi_{0}=\frac{\xi_{5}\left(\tau_{2}^{2}-4 \tau_{1} \tau_{3}\right)\left(2 \tau_{2}^{3}-9 \tau_{1} \tau_{2} \tau_{3}+2 \sqrt{\left(\tau_{2}^{2}-3 \tau_{1} \tau_{3}\right)^{3}}\right)}{81 \tau_{3}^{5}} \\
& \xi_{1}=-\frac{\xi_{5}\left(4 \tau_{2}^{4}+9 \tau_{1} \tau_{2}^{2} \tau_{3}-108 \tau_{1}^{2} \tau_{3}^{2}+4 \tau_{2} \sqrt{\left(\tau_{2}^{2}-3 \tau_{1} \tau_{3}\right)^{3}}\right)}{81 \tau_{3}^{4}}, \\
& \xi_{2}=\frac{\xi_{5}\left(-11 \tau_{2}^{3}+63 \tau_{1} \tau_{2} \tau_{3}-2 \sqrt{\left(\tau_{2}^{2}-3 \tau_{1} \tau_{3}\right)^{3}}\right)}{27 \tau_{3}^{3}}, \\
& \xi_{3}=\frac{\xi_{5}\left(\tau_{2}^{2}+7 \tau_{1} \tau_{3}\right)}{3 \tau_{3}^{2}}, \quad \xi_{4}=\frac{5 \xi_{5} \tau_{2}}{3 \tau_{3}} \\
& \zeta_{0}=-\frac{9 b n(m+1)(m+n+1) \xi_{5}}{2 a \tau_{3}(m-n+1)^{2}}, \\
& \tau_{0}=-\frac{2 \tau_{2}^{3}-9 \tau_{1} \tau_{2} \tau_{3}+2 \sqrt{\left(\tau_{2}^{2}-3 \tau_{1} \tau_{3}\right)^{3}}}{27 \tau_{3}^{2}}, \\
& \tau_{1}=\tau_{1}, \quad \tau_{2}=\tau_{2}, \\
& \tau_{3}=\tau_{3}, \quad \xi_{5}=\xi_{5}, \\
& c=-\frac{8 a n \sqrt{\left(\tau_{2}^{2}-3 \tau_{1} \tau_{3}\right)^{3}}}{27(m+1)(m+n+1) \tau_{3}^{2}} \text {. }
\end{aligned}
$$

Substituting these results into (6) and (10), we get

$$
\begin{aligned}
& \pm\left(\eta-\eta_{0}\right) \\
& =3 A_{2} \int((d \Gamma) \\
& \quad \times\left(\Gamma^{5}+\frac{\xi_{4}}{\xi_{5}} \Gamma^{4}+\frac{\xi_{3}}{\xi_{5}} \Gamma^{3}\right. \\
& \left.\left.\quad+\frac{\xi_{2}}{\xi_{5}} \Gamma^{2}+\frac{\xi_{1}}{\xi_{5}} \Gamma+\frac{\xi_{0}}{\xi_{5}}\right)^{-1 / 2}\right),
\end{aligned}
$$

where $A_{2}=\sqrt{-b n(1+m)(1+m+n) / 2 a \tau_{3}(1+m-n)^{2}}$. Integrating (38), we obtain the solutions to (1) as follows:

$$
\begin{aligned}
& \pm\left(\eta-\eta_{0}\right)=-\frac{2 A_{2}}{\sqrt{\left(\Gamma-\alpha_{1}\right)^{3}}}, \\
& \pm\left(\eta-\eta_{0}\right)=\frac{3 A_{2} \operatorname{arctanh}\left[\sqrt{\left(\Gamma-\alpha_{2}\right) /\left(\alpha_{1}-\alpha_{2}\right)}\right]}{\left(\alpha_{1}-\alpha_{2}\right)^{3 / 2}} \\
& -\frac{3 A_{2} \sqrt{\Gamma-\alpha_{2}}}{\left(\alpha_{1}-\alpha_{2}\right)\left(\Gamma-\alpha_{1}\right)}, \quad \alpha_{1}>\alpha_{2} \text {, } \\
& \pm\left(\eta-\eta_{0}\right)=-\frac{6 A_{2} \arctan \left[\sqrt{\left(\Gamma-\alpha_{1}\right) /\left(\alpha_{1}-\alpha_{2}\right)}\right]}{\left(\alpha_{1}-\alpha_{2}\right)^{3 / 2}} \\
& -\frac{6 A_{2}}{\sqrt{\Gamma-\alpha_{1}}\left(\alpha_{1}-\alpha_{2}\right)} \text {, } \\
& \pm\left(\eta-\eta_{0}\right)=\frac{6 A_{2} \operatorname{arctanh}\left[\sqrt{\left(\Gamma-\alpha_{3}\right) /\left(\alpha_{2}-\alpha_{3}\right)}\right]}{\alpha_{1}-\alpha_{2}} \\
& \times\left(\frac{1}{\sqrt{\alpha_{2}-\alpha_{3}}}-\frac{1}{\sqrt{\alpha_{1}-\alpha_{3}}}\right), \\
& \alpha_{1}>\alpha_{2}>\alpha_{3} \text {, } \\
& \pm\left(\eta-\eta_{0}\right) \\
& =\frac{-6 A_{2}}{\sqrt{\Gamma-\alpha_{1}}\left(\alpha_{1}-\alpha_{2}\right)\left(\alpha_{1}-\alpha_{3}\right)} \\
& \times\left[\sqrt{\left(\Gamma-\alpha_{2}\right)\left(\Gamma-\alpha_{3}\right)}+i(E(\varphi, l)-F(\varphi, l))\right],
\end{aligned}
$$

where

$$
\begin{gathered}
E(\varphi, l)=\int_{0}^{\varphi} \sqrt{1-l^{2} \sin ^{2} \psi} d \psi, \\
\varphi_{2}=-\arcsin \sqrt{\frac{\Gamma-\alpha_{1}}{\alpha_{2}-\alpha_{1}}}, \\
l_{2}^{2}=\frac{\alpha_{1}-\alpha_{2}}{\alpha_{1}-\alpha_{3}},
\end{gathered}
$$

$$
\begin{array}{r} 
\pm\left(\eta-\eta_{0}\right) \\
=\frac{-6 i A_{2}}{\sqrt{\alpha_{2}-\alpha_{3}}\left(\alpha_{1}-\alpha_{2}\right)}(F(\varphi, l)-\pi(\varphi, n, l)), \\
\alpha_{1}>\alpha_{2}>\alpha_{3}>\alpha_{4},
\end{array}
$$

where

$$
\begin{gathered}
\varphi_{3}=-\arcsin \sqrt{\frac{\alpha_{3}-\alpha_{2}}{\Gamma-\alpha_{2}}}, \quad l_{3}^{2}=\frac{\alpha_{2}-\alpha_{4}}{\alpha_{2}-\alpha_{3}}, \\
n=\frac{\alpha_{2}-\alpha_{1}}{\alpha_{2}-\alpha_{3}} .
\end{gathered}
$$


Also $\alpha_{1}, \alpha_{2}, \alpha_{3}, \alpha_{4}$, and $\alpha_{5}$ are the roots of the polynomial equation

$$
\Gamma^{5}+\frac{\xi_{4}}{\xi_{5}} \Gamma^{4}+\frac{\xi_{3}}{\xi_{5}} \Gamma^{3}+\frac{\xi_{2}}{\xi_{5}} \Gamma^{2}+\frac{\xi_{1}}{\xi_{5}} \Gamma+\frac{\xi_{0}}{\xi_{5}}=0 .
$$

Case 4. If we take $\epsilon=1, \delta=1$, and $\theta=4$, then

$$
\left(v^{\prime}\right)^{2}=\frac{\tau_{1}^{2}\left(\xi_{4} \Gamma^{4}+\xi_{3} \Gamma^{3}+\xi_{2} \Gamma^{2}+\xi_{1} \Gamma+\xi_{0}\right)}{\zeta_{0}+\zeta_{1} \Gamma},
$$

where $\xi_{4} \neq 0$ and $\zeta_{1} \neq 0$. Respectively, solving the algebraic equation system (9) yields

$$
\begin{aligned}
\xi_{0}= & \frac{\zeta_{0} \tau_{0}^{2}\left(M+2 a(1+m-n)^{2}\left(2 \zeta_{1} \tau_{0}+\zeta_{0} \tau_{1}\right)\right)}{b n(1+m)(1+m+n) \zeta_{1} \tau_{1}^{2}}, \\
\xi_{3}= & \xi_{3}, \\
\xi_{4}= & -\frac{2 a(1+m-n)^{2} \zeta_{1} \tau_{1}}{b n(1+m)(1+m+n)}, \\
\xi_{1}= & \left(\tau _ { 0 } \left(4 a(1+m-n)^{2} \zeta_{1}^{2} \tau_{0}^{2}\right.\right. \\
& +2 \zeta_{0} \tau_{1}\left(M+2 a(1+m-n)^{2} \zeta_{0} \tau_{1}\right) \\
& \left.\left.+\zeta_{1} \tau_{0}\left(M+8 a(1+m-n)^{2} \zeta_{0} \tau_{1}\right)\right)\right) \\
& \times\left(b n(1+m)(1+m+n) \zeta_{1} \tau_{1}^{2}\right)^{-1}, \\
\xi_{2}= & \left(6 a(1+m-n)^{2} \zeta_{1}^{2} \tau_{0}^{2}\right. \\
& +2 \zeta_{1} \tau_{0}\left(M+2 a(1+m-n)^{2} \zeta_{0} \tau_{1}\right) \\
& \left.+\zeta_{0} \tau_{1}\left(M+2 a(1+m-n)^{2} \zeta_{0} \tau_{1}\right)\right) \\
& \times\left(b n(1+m)(1+m+n) \zeta_{1} \tau_{1}^{2}\right)^{-1}, \\
\tau_{0}= & \zeta_{0}, \quad \zeta_{1}=\zeta_{1}, \\
& n\left(M+2 a(1+m-n)^{2}\left(3 \zeta_{1} \tau_{0}+\zeta_{0} \tau_{1}\right)\right) \\
& (1+m)(1+m+n)(1+m-n)^{2} \zeta_{1}
\end{aligned}
$$

where $M=b n(1+m)(1+m+n) \xi_{3}$. Substituting these results into (6) and (10), we get

$$
\begin{aligned}
& \pm\left(\eta-\eta_{0}\right) \\
& =A_{3} \int\left(\left(\Gamma+\frac{\zeta_{0}}{\zeta_{1}}\right)\right. \\
& \quad \times\left(\Gamma^{4}+\left(\frac{\xi_{3}}{\xi_{4}}\right) \Gamma^{3}+\left(\frac{\xi_{2}}{\xi_{4}}\right) \Gamma^{2}\right. \\
& \left.\left.+\left(\frac{\xi_{1}}{\xi_{4}}\right) \Gamma+\left(\frac{\xi_{0}}{\xi_{4}}\right)\right)^{-1}\right)^{1 / 2} d \Gamma,
\end{aligned}
$$

where $A_{3}=\sqrt{b n(1+m)(1+m+n) / 2 a \tau_{1}(1+m-n)^{2}}$. Integrating (45), we obtain the solution to (1) as follows:

$$
\begin{aligned}
& \pm\left(\eta-\eta_{0}\right)=-A_{3} \sqrt{\frac{\zeta_{1}}{\zeta_{0}+\zeta_{1} \alpha_{1}}} \\
& \times \operatorname{arctanh}\left[\sqrt{\frac{\zeta_{0}+\zeta_{1} \Gamma}{\zeta_{0}+\zeta_{1} \alpha_{1}}}\right] \\
& -\frac{A_{3}}{\Gamma-\alpha_{1}} \sqrt{\frac{\zeta_{0}+\zeta_{1} \Gamma}{\zeta_{1}}} \text {, } \\
& \pm\left(\eta-\eta_{0}\right)=\frac{2 A_{3}}{\alpha_{1}-\alpha_{2}} \\
& \times\left(-\sqrt{\frac{\zeta_{0}+\zeta_{1} \alpha_{1}}{\zeta_{1}}}\right. \\
& \times \operatorname{arctanh}\left[\sqrt{\frac{\zeta_{0}+\zeta_{1} \Gamma}{\zeta_{0}+\zeta_{1} \alpha_{1}}}\right] \\
& +\sqrt{\zeta_{0}+\zeta_{1} \alpha_{2}} \\
& \left.\times \operatorname{arctanh}\left[\sqrt{\frac{\zeta_{0}+\zeta_{1} \Gamma}{\zeta_{0}+\zeta_{1} \alpha_{2}}}\right]\right), \\
& \pm\left(\eta-\eta_{0}\right)=2 A_{3} \\
& \times\left(\sqrt{\frac{\left(\Gamma-\alpha_{1}\right)\left(\zeta_{0}+\zeta_{1}\right.}{\zeta_{1}\left(\Gamma-\alpha_{2}\right)^{2}}}\right. \\
& \left.+i \sqrt{\alpha_{1}-\alpha_{2}}(E(\varphi, l)-F(\varphi, l))\right),
\end{aligned}
$$

where

$$
\begin{gathered}
\varphi_{4}=-\arcsin \sqrt{\frac{\zeta_{1}\left(\alpha_{1}-\Gamma\right)}{\zeta_{0}+\zeta_{1} \alpha_{1}},} \\
l_{4}^{2}=\frac{\zeta_{0}+\zeta_{1} \alpha_{1}}{\zeta_{1}\left(\alpha_{1}-\alpha_{2}\right)}, \\
\pm\left(\eta-\eta_{0}\right)=\frac{-2 A_{3}}{\sqrt{\alpha_{2}-\alpha_{1}}} E(\varphi, l),
\end{gathered}
$$

where

$$
\begin{aligned}
& \varphi_{5}=\arcsin \left[\sqrt{\frac{\alpha_{2}-\alpha_{1}}{\Gamma-\alpha_{1}}}\right], \\
& l_{5}^{2}=\frac{\zeta_{0}+\zeta_{1} \alpha_{1}}{\zeta_{1}\left(\alpha_{1}-\alpha_{2}\right)}, \\
& \pm\left(\eta-\eta_{0}\right)=\frac{-2 i A_{3}}{\left(\alpha_{1}-\alpha_{2}\right) \sqrt{\zeta_{1}\left(\zeta_{0}+\zeta_{1} \alpha_{2}\right)}}
\end{aligned}
$$


Abstract and Applied Analysis

9

$$
\begin{aligned}
\times & \left(\zeta_{0}(F(\varphi, l)-\pi(\varphi, n, l))\right. \\
& \left.+\zeta_{1}\left(\alpha_{2} F(\varphi, l)-\alpha_{2} \pi(\varphi, n, l)\right)\right),
\end{aligned}
$$

where

$$
\begin{aligned}
& \varphi_{6}=-\arcsin \sqrt{\frac{\zeta_{0}+\zeta_{1} \alpha_{2}}{\zeta_{1}\left(\alpha_{2}-\Gamma\right)}}, \\
& l_{6}^{2}=\frac{\zeta_{1}\left(\alpha_{2}-\alpha_{3}\right)}{\zeta_{0}+\zeta_{1} \alpha_{2}}, \quad n_{1}=\frac{\zeta_{1}\left(\alpha_{2}-\alpha_{1}\right)}{\zeta_{0}+\zeta_{1} \alpha_{2}} .
\end{aligned}
$$

Case 5. If we take $\epsilon=1, \delta=2$, and $\theta=5$, then

$$
\left(v^{\prime}\right)^{2}=\frac{\left(\tau_{1}+2 \tau_{2} \Gamma\right)^{2}\left(\xi_{5} \Gamma^{5}+\xi_{4} \Gamma^{4}+\xi_{3} \Gamma^{3}+\xi_{2} \Gamma^{2}+\xi_{1} \Gamma+\xi_{0}\right)}{\zeta_{0}+\zeta_{1} \Gamma},
$$

where $\xi_{5} \neq 0$ and $\zeta_{1} \neq 0$. Respectively, solving the algebraic equation system (9) yields

$$
\begin{gathered}
\xi_{0}=\frac{\tau_{0}^{2}\left(-2 \xi_{5} \tau_{1}+\xi_{4} \tau_{2}\right)}{\tau_{2}^{3}}, \\
\xi_{1}=\frac{\tau_{0}\left(2 \xi_{4} \tau_{1} \tau_{2}+\xi_{5}\left(-4 \tau_{1}^{2}+\tau_{0} \tau_{2}\right)\right)}{\tau_{2}^{3}}, \\
\xi_{2}=\frac{\xi_{4} \tau_{2}\left(\tau_{1}^{2}+2 \tau_{0} \tau_{2}\right)-2 \xi_{5}\left(\tau_{1}^{3}+\tau_{0} \tau_{1} \tau_{2}\right)}{\tau_{2}^{3}}, \\
\zeta_{0}=\frac{-2 b n(1+m)(1+m+n)\left(\xi_{4} \tau_{2}-2 \xi_{5} \tau_{1}\right)}{a(1+m-n)^{2} \tau_{2}^{2}}, \\
\xi_{3}=\frac{-3 \xi_{5} \tau_{1}^{2}+2 \tau_{2}\left(\xi_{5} \tau_{0}+\xi_{4} \tau_{1}\right)}{\tau_{2}^{2}}, \\
\zeta_{1}=\frac{-2 b n(1+m)(1+m+n) \xi_{5}}{a(1+m-n)^{2} \tau_{2}^{2}}, \\
\tau_{0}=\tau_{0}, \quad \frac{\xi_{1}=\xi_{5},}{2(1+m)(1+m+n) \tau_{2}} . \\
\tau_{4}=\tau_{4}, \\
a n\left(\tau_{1}^{2}-4 \tau_{0} \tau_{2}\right)
\end{gathered}
$$

Substituting these results into (6) and (10), we get

$$
\begin{aligned}
\pm\left(\eta-\eta_{0}\right)=A_{4} & \\
& \times \int\left(\left(\Gamma+\frac{\zeta_{0}}{\zeta_{1}}\right)\right. \\
& \times\left(\Gamma^{5}+\frac{\xi_{4}}{\xi_{5}} \Gamma^{4}+\frac{\xi_{3}}{\xi_{5}} \Gamma^{3}\right. \\
& \left.\left.+\frac{\xi_{2}}{\xi_{5}} \Gamma^{2}+\frac{\xi_{1}}{\xi_{5}} \Gamma+\frac{\xi_{0}}{\xi_{5}}\right)^{-1}\right)^{1 / 2} d \Gamma,
\end{aligned}
$$

where $A_{4}=\sqrt{-2 b n(1+m)(1+m+n) / a \tau_{2}^{2}(1+m-n)^{2}}$. Integrating (52), we obtain the solution to (1) as follows:

$$
\begin{aligned}
& \pm\left(\eta-\eta_{0}\right)=\frac{-2 A_{4}}{3 \sqrt{\zeta_{1}}\left(\zeta_{0}+\zeta_{1} \alpha_{1}\right)}\left(\frac{\zeta_{0}+\zeta_{1} \Gamma}{\Gamma-\alpha_{1}}\right)^{3 / 2}, \\
& \pm\left(\eta-\eta_{0}\right) \\
& =\frac{-A_{4}\left(\zeta_{0}+\zeta_{1} \alpha_{2}\right)}{2\left(\alpha_{1}-\alpha_{2}\right)^{3 / 2} \sqrt{\zeta_{1}\left(\zeta_{0}+\zeta_{1} \alpha_{1}\right)}} \\
& \times \ln \mid\left(\Gamma-\alpha_{1}\right) \\
& \times\left(\zeta_{0}\left(\Gamma+\alpha_{1}-2 \alpha_{2}\right)\right. \\
& +2 \sqrt{\left(\zeta_{0}+\zeta_{1} \Gamma\right)\left(\zeta_{0}+\zeta_{1} \alpha_{1}\right)\left(\Gamma-\alpha_{2}\right)\left(\alpha_{1}-\alpha_{2}\right)} \\
& \left.+\zeta_{1}\left(2 \Gamma \alpha_{1}-\alpha_{2}\left(\Gamma+\alpha_{1}\right)\right)\right)^{-1} \mid \\
& -\frac{A_{4}}{\left(\alpha_{1}-\alpha_{2}\right)\left(\Gamma-\alpha_{1}\right)} \sqrt{\frac{\left(\zeta_{0}+\zeta_{1} \Gamma\right)\left(\Gamma-\alpha_{2}\right)}{\zeta_{1}}}, \quad \alpha_{1}>\alpha_{2} \text {, } \\
& \pm\left(\eta-\eta_{0}\right) \\
& =\frac{-2 A_{4}}{\left(\alpha_{1}-\alpha_{2}\right)} \sqrt{\frac{\zeta_{0}+\zeta_{1} \Gamma}{\zeta_{1}\left(\Gamma-\alpha_{1}\right)}} \\
& -\frac{2 A_{4}}{\left(\alpha_{1}-\alpha_{2}\right)^{3 / 2}} \sqrt{\frac{\zeta_{0}+\zeta_{1} \alpha_{2}}{\zeta_{1}}} \\
& \times \arctan \left[\sqrt{\frac{\left(\Gamma-\alpha_{1}\right)\left(\zeta_{0}+\zeta_{1} \alpha_{2}\right)}{\left(\alpha_{1}-\alpha_{2}\right)\left(\zeta_{0}+\zeta_{1} \Gamma\right)}}\right], \\
& \pm\left(\eta-\eta_{0}\right) \\
& =\frac{-A_{4}}{\alpha_{1}-\alpha_{3}} \sqrt{\frac{\zeta_{0}+\zeta_{1} \alpha_{2}}{\zeta_{1}\left(\alpha_{2}-\alpha_{3}\right)}} \\
& \times \ln \mid\left(\alpha_{2}-\Gamma\right) \\
& \times\left(\zeta_{0}\left(\Gamma+\alpha_{2}-2 \alpha_{3}\right)\right. \\
& +2 \sqrt{\left(\zeta_{0}+\zeta_{1} \Gamma\right)\left(\zeta_{0}+\zeta_{1} \alpha_{2}\right)\left(\Gamma-\alpha_{3}\right)\left(\alpha_{2}-\alpha_{3}\right)} \\
& \left.+\zeta_{1}\left(2 \Gamma \alpha_{2}-\alpha_{3}\left(\Gamma+\alpha_{2}\right)\right)\right)^{-1} \\
& -\frac{A_{4}}{\alpha_{1}-\alpha_{3}} \\
& \times \sqrt{\frac{\zeta_{0}+\zeta_{1} \alpha_{1}}{\zeta_{1}\left(\alpha_{1}-\alpha_{3}\right)}} \\
& \times \ln \mid\left(\zeta_{0}\left(\Gamma+\alpha_{1}-2 \alpha_{3}\right)\right. \\
& +2 \sqrt{\left(\zeta_{0}+\zeta_{1} \Gamma\right)\left(\zeta_{0}+\zeta_{1} \alpha_{1}\right)\left(\Gamma-\alpha_{3}\right)\left(\alpha_{1}-\alpha_{3}\right)}
\end{aligned}
$$




$$
\begin{aligned}
&+\left.\zeta_{1}\left(2 \Gamma \alpha_{1}-\alpha_{3}\left(\Gamma+\alpha_{1}\right)\right)\right) \\
& \times\left(\Gamma-\alpha_{2}\right)^{-1} \mid, \quad \alpha_{1}>\alpha_{2}>\alpha_{3}, \\
& \pm\left(\eta-\eta_{0}\right)= \frac{-2 A_{4}}{\alpha_{1}-\alpha_{3}} \\
& \times \sqrt{\frac{\zeta_{0}+\zeta_{1} \alpha_{3}}{\zeta_{1}\left(\alpha_{1}-\alpha_{2}\right)}} E(\varphi, l), \\
& \alpha_{1}>\alpha_{2}>\alpha_{3},
\end{aligned}
$$

where

$$
\begin{aligned}
\varphi_{7}= & \arcsin \sqrt{\frac{\left(\Gamma-\alpha_{3}\right)\left(\alpha_{2}-\alpha_{1}\right)}{\left(\Gamma-\alpha_{1}\right)\left(\alpha_{2}-\alpha_{3}\right)},} \\
l_{7}^{2}= & \frac{\left(\alpha_{3}-\alpha_{2}\right)\left(\zeta_{0}+\zeta_{1} \alpha_{1}\right)}{\left(\alpha_{1}-\alpha_{2}\right)\left(\zeta_{0}+\zeta_{1} \alpha_{3}\right)}, \\
\pm & \left(\eta-\eta_{0}\right) \\
= & \frac{2 A_{4}\left(\alpha_{2}-\alpha_{4}\right)}{\left(\alpha_{1}-\alpha_{2}\right)\left(\alpha_{3}-\alpha_{4}\right) \sqrt{\zeta_{1}\left(\alpha_{2}-\alpha_{3}\right)\left(\zeta_{0}+\zeta_{1} \alpha_{4}\right)}} \\
& \times\left(\frac{\left(\zeta_{0}+\zeta_{1} \Gamma\right)\left(\alpha_{3}-\alpha_{4}\right)}{\alpha_{1}-\alpha_{2}} \pi(\varphi, n, l)\right. \\
& \left.+\frac{\left(\zeta_{0}+\zeta_{1} \alpha_{2}\right)\left(\alpha_{4}-\alpha_{3}\right)}{\alpha_{2}-\alpha_{4}} F(\varphi, l)\right),
\end{aligned}
$$

where

$$
\begin{gathered}
\varphi_{8}=\arcsin \sqrt{\frac{\left(\Gamma-\alpha_{3}\right)\left(\alpha_{2}-\alpha_{1}\right)}{\left(\Gamma-\alpha_{1}\right)\left(\alpha_{2}-\alpha_{3}\right)}}, \\
l_{8}^{2}=\frac{\left(\alpha_{3}-\alpha_{2}\right)\left(\zeta_{0}+\zeta_{1} \alpha_{1}\right)}{\left(\alpha_{1}-\alpha_{2}\right)\left(\zeta_{0}+\zeta_{1} \alpha_{3}\right)}, \\
n_{2}=-\frac{\left(\alpha_{1}-\alpha_{2}\right)\left(\alpha_{3}-\alpha_{4}\right)}{\left(\alpha_{2}-\alpha_{3}\right)\left(\alpha_{1}-\alpha_{4}\right)}, \\
\alpha_{1}>\alpha_{2}>\alpha_{3}>\alpha_{4} .
\end{gathered}
$$

\section{Discussion}

Thus we introduce a more general extended trial equation method for nonlinear partial differential equations as follows.

Step 1. Extended trial equation (6) can be reduced to the following more general form:

$$
u=\frac{A(\Gamma)}{B(\Gamma)}=\frac{\sum_{i=0}^{\delta} \tau_{i} \Gamma^{i}}{\sum_{j=0}^{\mu} \omega_{j} \Gamma^{j}},
$$

where

$$
\left(\Gamma^{\prime}\right)^{2}=\Lambda(\Gamma)=\frac{\Phi(\Gamma)}{\Psi(\Gamma)}=\frac{\xi_{\theta} \Gamma^{\theta}+\cdots+\xi_{1} \Gamma+\xi_{0}}{\zeta_{\epsilon} \Gamma^{\epsilon}+\cdots+\zeta_{1} \Gamma+\zeta_{0}} .
$$

Here, $\tau_{i}(i=0, \ldots, \delta), \omega_{j}(j=0, \ldots, \mu), \xi_{\varsigma}(\varsigma=0, \ldots, \theta)$, and $\zeta_{\sigma}(\sigma=0, \ldots, \epsilon)$ are the constants to be specified.

Step 2. Taking trial equations (56) and (57), we derive the following equations:

$$
\begin{aligned}
\left(u^{\prime}\right)^{2}=\frac{\Phi(\Gamma)}{\Psi(\Gamma)} \frac{\left(A^{\prime}(\Gamma) B(\Gamma)-A(\Gamma) B^{\prime}(\Gamma)\right)^{2}}{B^{4}(\Gamma)}, \\
u^{\prime \prime}=\left(A^{\prime}(\Gamma) B(\Gamma)-A(\Gamma) B^{\prime}(\Gamma)\right) \\
\times\left\{\left(\Phi^{\prime}(\Gamma) \Psi(\Gamma)-\Phi(\Gamma) \Psi^{\prime}(\Gamma)\right) B(\Gamma)\right. \\
\left.\quad-4 \Phi(\Gamma) \Psi(\Gamma) B^{\prime}(\Gamma)\right\} \\
+2 \Phi(\Gamma) \Psi(\Gamma) B(\Gamma)\left(A^{\prime \prime}(\Gamma) B(\Gamma)-A(\Gamma) B^{\prime \prime}(\Gamma)\right) \\
\times\left(2 B^{3}(\Gamma) \Psi^{2}(\Gamma)\right)^{-1}
\end{aligned}
$$

and other derivation terms such as $u^{\prime \prime \prime}$.

Step 3. Substituting $u^{\prime}, u^{\prime \prime}$, and other derivation terms into (5) yields the following equation:

$$
\Omega(\Gamma)=\varrho_{s} \Gamma^{\mathcal{s}}+\cdots+\varrho_{1} \Gamma+\varrho_{0}=0 .
$$

According to the balance principle we can determine a relation of $\theta, \epsilon, \delta$, and $\mu$.

Step 4. Letting the coefficients of $\Omega(\Gamma)$ all be zero will yield an algebraic equations system $\varrho_{i}=0 \quad(i=0, \ldots, s)$. Solving this equations system, we will determine the values $\tau_{0}, \ldots \tau_{\delta}$; $\omega_{0}, \ldots, \omega_{\mu} ; \xi_{0}, \ldots, \xi_{\theta} ;$ and $\zeta_{0}, \ldots, \zeta_{\epsilon}$.

Step 5. Substituting the results obtained in Step 4 into (57) and integrating (57), we can find the exact solutions of (3).

\section{Conclusions and Remarks}

In this study, we proposed an extended trial equation method and used it to obtain some soliton and elliptic function solutions to the generalized $K(m, n)$ equation. Otherwise, we discussed a more general trial equation method. The proposed method can also be applied to other nonlinear differential equations with nonlinear evolution.

\section{References}

[1] R. Hirota, "Exact solutions of the Korteweg-de-Vries equation for multiple collisions of solitons," Physics Letters A, vol. 27, pp. 1192-1194, 1971.

[2] W. Malfliet and W. Hereman, "The tanh method: exact solutions of nonlinear evolution and wave equations," Physica Scripta, vol. 54, no. 6, pp. 563-568, 1996.

[3] M. Naja, S. Arbabi, and M. Naja, "New application of sinecosine method for the generalized $(2+1)$-dimensional nonlinear evolution equations," International Journal of Advanced Mathematical Sciences, vol. 1, no. 2, pp. 45-49, 2013. 
[4] Y. Gurefe, A. Sonmezoglu, and E. Misirli, "Application of the trial equation method for solving some nonlinear evolution equations arising in mathematical physics," Pramana Journal of Physics, vol. 77, pp. 1023-1029, 2011.

[5] Y. Gurefe, A. Sonmezoglu, and E. Misirli, "Application of an irrational trial equation method to high-dimensional nonlinear evolution equations," Journal of Advanced Mathematical Studies, vol. 5, no. 1, pp. 41-47, 2012.

[6] C. S. Liu, "Trial equation method and its applications to nonlinear evolution equations," Acta Physica Sinica, vol. 54, no. 6, pp. 2505-2509, 2005.

[7] C. S. Liu, "Trial equation method for nonlinear evolution equations with rank inhomogeneous: mathematical discussions and applications," Communications in Theoretical Physics, vol. 45, pp. 219-223, 2006.

[8] Y. Pandir, Y. Gurefe, U. Kadak, and E. Misirli, "Classification of exact solutions for some nonlinear partial differential equations with generalized evolution," Abstract and Applied Analysis, vol. 2012, Article ID 478531, 16 pages, 2012.

[9] Y. Gurefe, E. Misirli, A. Sonmezoglu, and M. Ekici, "Extended trial equation method to generalized partial differential equations," Applied Mathematics and Computation, vol. 219, no. 10, pp. 5253-5260, 2013.

[10] Y. Pandir, Y. Gurefe, and E. Misirli, "Classification of exact solutions to the generalized KadomtsevPetviashvili equation," Physica Scripta, vol. 87, Article ID 025003, 12 pages, 2013.

[11] Y. Pandir, Y. Gurefe, and E. Misirli, "The extended trial equation method for some time fractional differential equations," Discrete Dynamics in Nature and Society, vol. 2013, Article ID 491359, 13 pages, 2013.

[12] C. S. Liu, "A new trial equation method and its applications," Communications in Theoretical Physics, vol. 45, pp. 395-397, 2006.

[13] C. Y. Jun, "Classification of traveling wave solutions to the Vakhnenko equations," Computational and Applied Mathematics, vol. 62, no. 10, pp. 3987-3996, 2011.

[14] C. Y. Jun, "Classification of traveling wave solutions to the modified form of the Degasperis-Procesi equation," Mathematical and Computer Modelling, vol. 56, no. 1-2, pp. 43-48, 2012.

[15] C.-S. Liu, "Applications of complete discrimination system for polynomial for classifications of traveling wave solutions to nonlinear differential equations," Computer Physics Communications, vol. 181, no. 2, pp. 317-324, 2010.

[16] G. Ebadi and A. Biswas, "The G'/G method and topological soliton solution of the $\mathrm{K}(\mathrm{m}, \mathrm{n})$ equation," Communications in Nonlinear Science and Numerical Simulation, vol. 16, no. 6, pp. 2377-2382, 2011.

[17] M. S. Bruzon and M. L. Gandarias, "Classical potential symmetries of the $\mathrm{K}(\mathrm{m}, \mathrm{n})$ equation with generalized evolution term," WSEAS Transactions on Mathematics, vol. 9, no. 4, pp. 275-284, 2010.

[18] M. S. Bruzon, M. L. Gandarias, G. A. Gonzalez, and R. Hansen, "The $\mathrm{K}(\mathrm{m}, \mathrm{n})$ equation with generalized evolution term studied by symmetry reductions and qualitative analysis," Applied Mathematics and Computation, vol. 218, no. 20, pp. 10094-10105, 2012. 


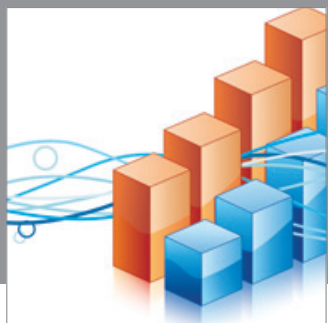

Advances in

Operations Research

mansans

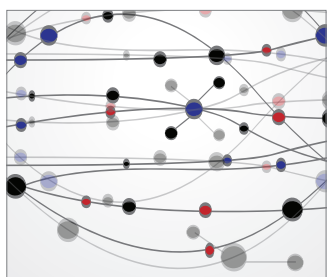

The Scientific World Journal
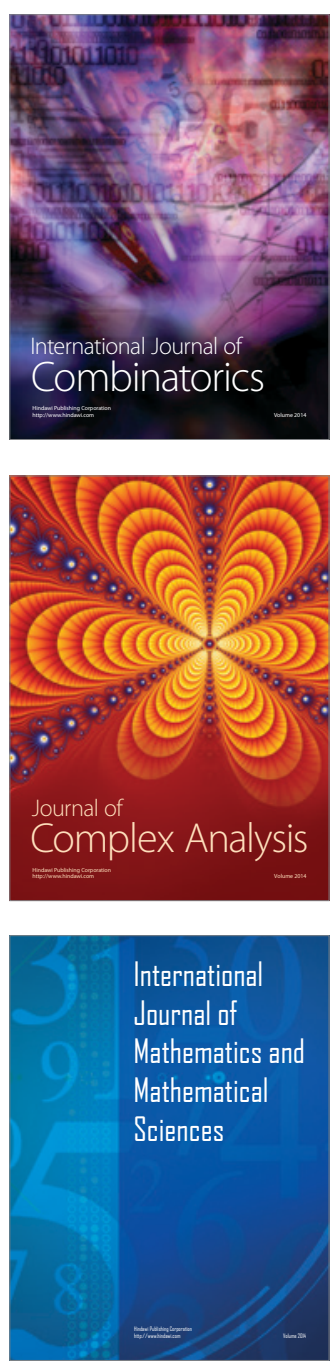
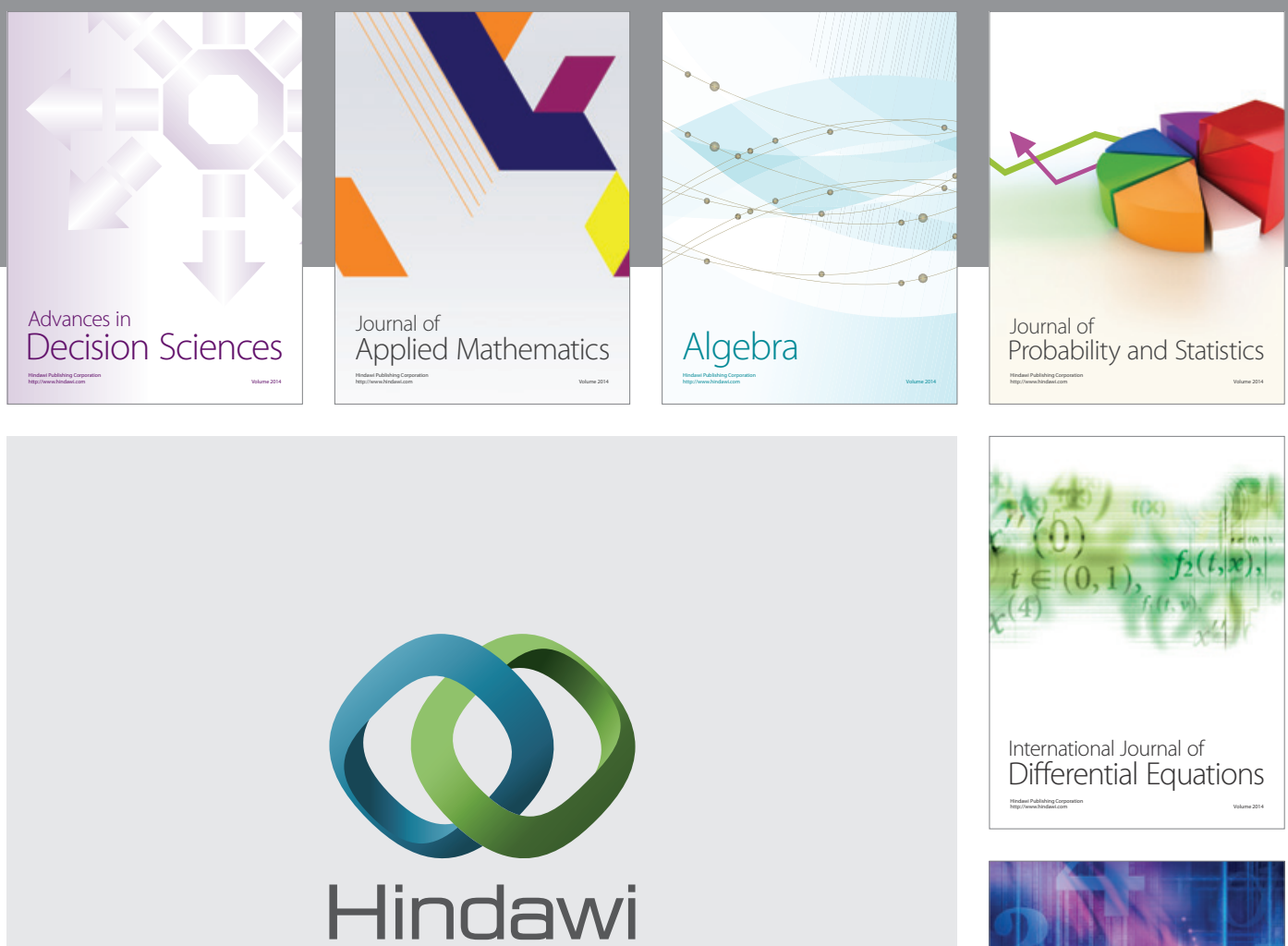

Submit your manuscripts at http://www.hindawi.com
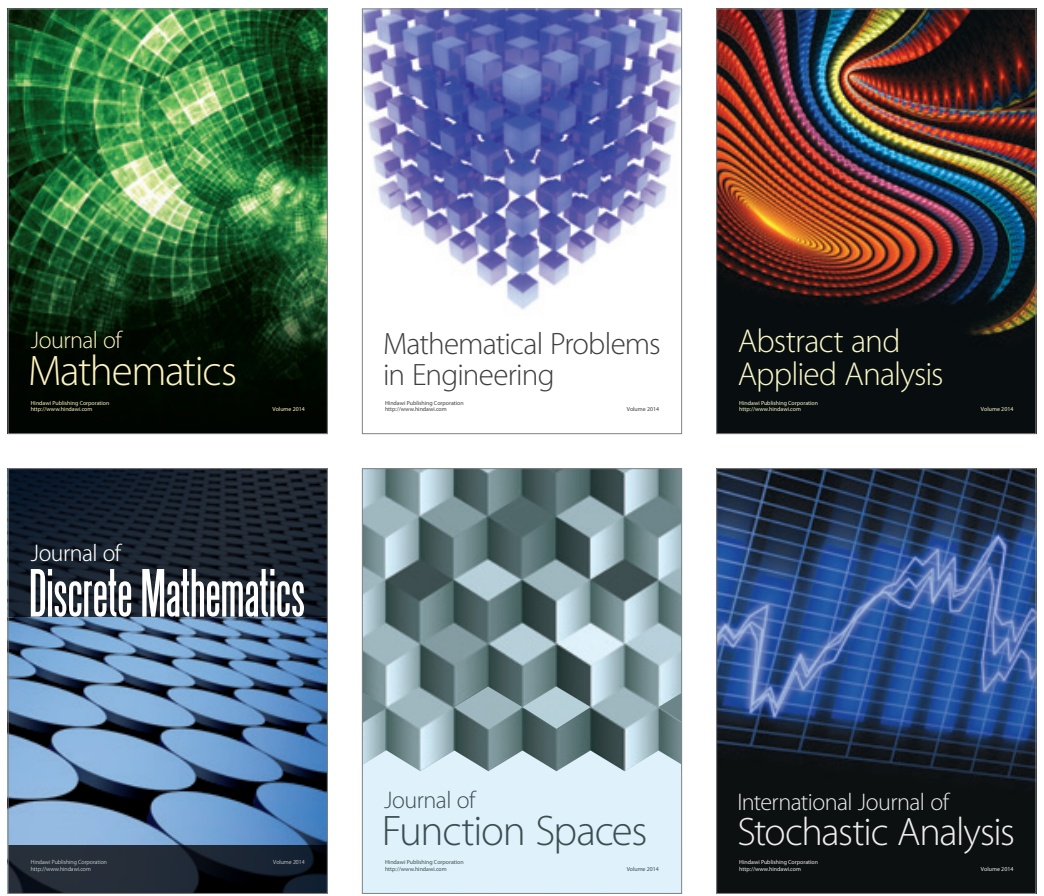

Journal of

Function Spaces

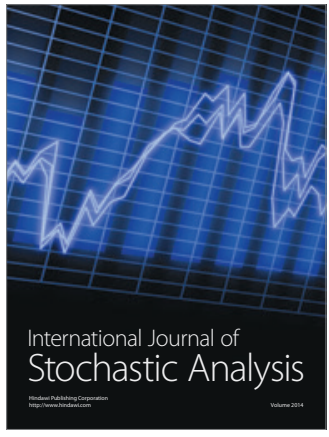

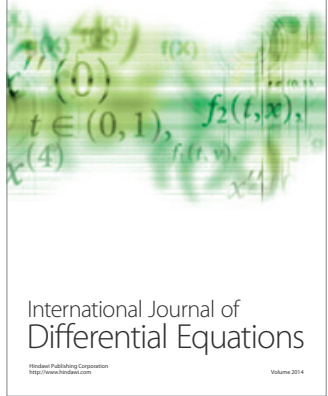
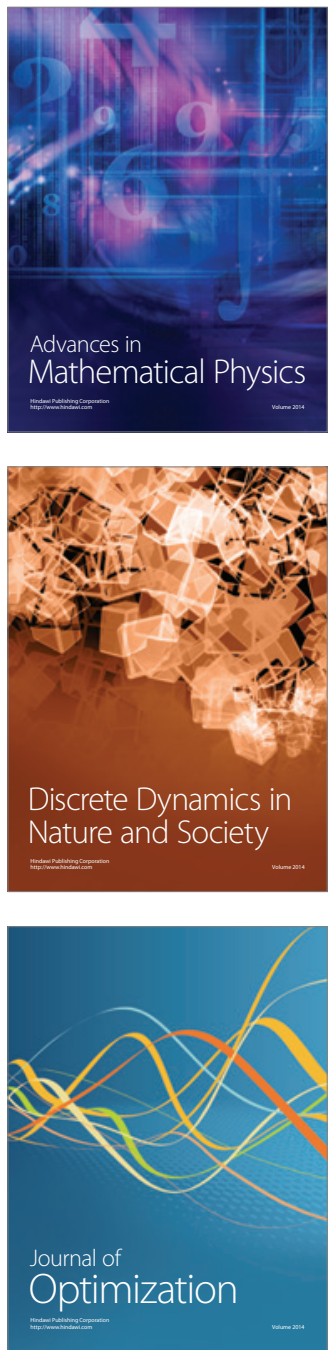